\section{References}

1. Oostindie SC, van der Horst HJ, Lindorfer MA, et al. CD20 and CD37 antibodies synergize to activate complement by Fc-mediated clustering. Haematologica. 2019;104(9):1841-1852.

2. Diebolder CA, Beurskens FJ, de Jong RN, et al. Complement is activated by $\operatorname{IgG}$ hexamers assembled at the cell surface. Science. 2014;343(6176):1260-1263.

3. Salles G, Barrett M, Foa R, et al. Rituximab in B-cell hematologic malignancies: a review of 20 years of clinical experience. Adv Ther. 2017;34(10):2232-2273.

4. Zent CS, Victoria Wang X, Ketterling RP, et al. A phase II randomized trial comparing standard and low dose rituximab combined with alemtuzumab as initial treatment of progressive chronic lymphocytic leukemia in older patients: a trial of the ECOG-ACRIN cancer research group (E1908). Am J Hematol. 2016;91(3):308-312.

5. Witkowska M, Smolewski P, Robak T. Investigational therapies targeting CD37 for the treatment of B-cell lymphoid malignancies. Expert Opin Investig Drugs. 2018;27(2):171-177.

6. Yeung L, Hickey MJ, Wright MD. The many and varied roles of tetraspanins in immune cell recruitment and migration. Front Immunol. 2018;9:1644.

7. van Spriel AB, de Keijzer S, van der Schaaf A, et al. The tetraspanin CD37 orchestrates the alpha(4)beta(1) integrin-Akt signaling axis and supports long-lived plasma cell survival. Sci Signal. 2012:5(250):ra82.

8. Byrd JC, Pagel JM, Awan FT, et al. A phase 1 study evaluating the safety and tolerability of otlertuzumab, an anti-CD37 mono-specific ADAPTIR therapeutic protein in chronic lymphocytic leukemia. Blood. 2014;123(9):1302-1308.
9. Robak T, Hellmann A, Kloczko J, et al. Randomized phase 2 study of otlertuzumab and bendamustine versus bendamustine in patients with relapsed chronic lymphocytic leukaemia. Br J Haematol 2017;176(4):618-628

10. Stilgenbauer S, Aurran Schleinitz T, Eichhorst B, et al. Phase 1 firstin-human trial of the anti-CD37 antibody BI 836826 in relapsed/refractory chronic lymphocytic leukemia. Leukemia. 2019 May 14. [Epub ahead of print]

11. Weiner LM, Surana R, Wang S. Monoclonal antibodies: versatile platforms for cancer immunotherapy. Nat Rev Immunol. 2010;10(5):317-327.

12. Saxena A, Wu D. Advances in therapeutic Fc engineering - modulation of IgG-associated effector functions and serum half-life. Front Immunol. 2016;7:580.

13. de Jong RN, Beurskens FJ, Verploegen S, et al. A novel platform for the potentiation of therapeutic antibodies based on antigen-dependent formation of $\operatorname{IgG}$ hexamers at the cell surface. PLoS Biol. 2016;14(1):e1002344.

14. Cook EM, Lindorfer MA, van der Horst $\mathrm{H}$, et al. Antibodies that efficiently form hexamers upon antigen binding can induce complement-dependent ctotoxicity under complement-limiting conditions. J Immunol. 2016;197(5):1762-1775

15. Labrijn AF, Janmaat ML, Reichert JM, Parren P. Bispecific antibodies: a mechanistic review of the pipeline. Nat Rev Drug Discov. 2019 Jun 7. [Epub ahead of print]

16. Haurum JS. Recombinant polyclonal antibodies: the next generation of antibody therapeutics? Drug Discov Today. 2006;11(13-14):655660.

17. Taylor RP, Lindorfer MA. Fcgamma-receptor-mediated trogocytosis impacts $\mathrm{mAb}$-based therapies: historical precedence and recent developments. Blood. 2015;125(5):762-766.

\title{
The secret afterlife of platelets
}

\begin{abstract}
Nicholas A. Arce ${ }^{1,2}$ and Renhao $\mathrm{Li}^{1}$
'Aflac Cancer and Blood Disorders Center, Children's Healthcare of Atlanta, Department of Pediatrics, Emory University School of Medicine and 'Graduate Program of Molecular and Systems Pharmacology, Graduate Division of Biological and Biomedical Sciences, Emory University, Atlanta, GA, USA
\end{abstract}

E-mail: RENHAO LI - renhao.li@emory.edu

doi:10.3324/haematol.2019.224170

$\mathrm{P}$ atelets express a wide variety of receptors and signaling molecules that enable responses to diverse physiological and pathological stimulants. For instance, in normal hemostasis, exposure of subendothelial collagen may elicit platelet activation at the site of injury via glycoprotein (GP)VI, integrin $\alpha_{2} \beta_{1}$, and, through plasma von Willebrand factor, the GPIb-IX-V complex. Moreover, GPIb-IX-V in tandem with protease-activated receptors mediate thrombin-induced platelet signaling and activation. GPIba serves as a receptor for low concentrations of thrombin, transmitting a mechanosensory signal to mediate calcium-dependent 14-3-3 signaling while GPIb-IXdependent Rac1/LIMK1 signaling is modulated by protease-activated receptors. ${ }^{1,2}$ Upon activation, platelets aggregate and form clots that are interwoven with fibrin strands. Over the last several decades, much of the research effort has been focused on how platelets are rapidly activated by various agonists via their respective receptors and how activating, and sometimes inhibitory, signals amplify and propagate in the platelet. In most of these studies, the investigation ends at the cessation of blood flow, the formation of the clot, and/or the appearance of molecular signs that are well associated with platelet activation. A few minutes following platelet activation and aggregation, the blood clot contracts. In studies of clot contraction, the investigation often ends at the shrinkage of the platelet clot. ${ }^{3}$ However, little is known about the platelets in the clot following the contraction of the platelet/fibrin clot. In other words, after the formation of a stable blood clot, where do platelets go?

A study by Kim et al., published in this issue of Haematologica, demonstrates that after activation and contraction, thrombin-stimulated platelets break up into membrane particles, in a process termed platelet fragmentation. ${ }^{4}$ Thrombin is a major nexus between coagulation and platelet activation, as it generates fibrin to form a crosslinked fibrin plug and concurrently activates aforementioned receptors on the platelet surface. ${ }^{5}$ Platelet vesiculation and/or microparticle formation has been previously observed in response to thrombin and thrombin receptor activating peptide. ${ }^{6-8}$ The role that these platelet fragments play in hemostasis or platelet clearance has yet to be elucidated. In this new study, interestingly, Kim et al. observed a bimodal distribution of platelet fragments, the size of which can be attributed to the origin of the fragment. Filopodia as well as the main platelet body are two sources of platelet fragmentation, as smaller fragments were generated by filopodia, and larger fragments were generated from the cell body. Thus, it appears that platelet breakdown in response to thrombin stimulation is a regulated process of drastic morphological changes, platelet fragmentation, loss of function, and metabolic exhaustion. Platelet fragmentation may be a relatively newly discovered platelet behavior, adding to the ever-growing list of what 


\section{Thrombin \\ 1 \\ Platelet surface receptor

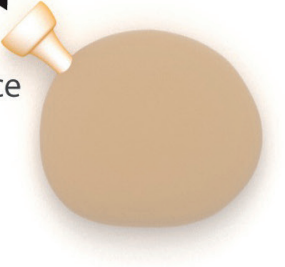 \\ Platelet

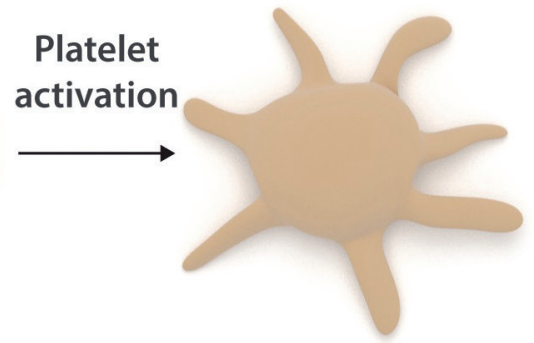 \\ Activated platelet
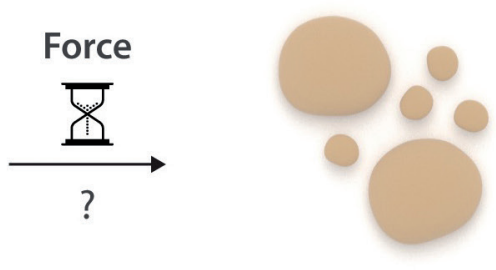 \\ Fragmentation of platelet}

Figure 1. An illustration of thrombin-induced effects on platelets over time. Thrombin produced through coagulation binds to and activates platelet receptors, leading to platelet activation, degranulation, and filopodia formation. At a certain time after the hemostatic plug has contracted, platelets break down into membranebound fragments of defined sizes through a distinct mechanism.

happens to platelets after activation, and how they may play a role in hemostasis or clearance. This paradigm shift may help to elucidate novel mechanisms of platelet behavior and clarify the functional roles of such fragments of previously activated platelets.

Using transmission and scanning electron microscopy, Kim et al. observed that after 15 min of thrombin treatment, platelets broke up into separate membrane-bound particulates that contained granules, mitochondria, and vacuoles. Remarkably, this fragmentation was not seen after exposure to other platelet agonists such as collagen or ADP, although the platelets exhibited morphological changes associated with activation such as filopodia formation and spreading. This finding suggests that platelet activation creates agonist-specific behavior, and only when thrombin is being generated can platelets start to break up into fragments.

As actin is necessary for cytoskeletal rearrangement and filopodia extension, ${ }^{9}$ the authors investigated localization of actin after exposure to thrombin. After fragmentation, actin was retained in the particulates, and gradually disappeared as the fragments became smaller. It is also worthy of note that intracellular levels of calcium correlated with fragmentation, such that calcium levels dropped as the platelets disintegrated. Mitochondrial function also decreased as the platelets fragmented. Mitochondria appeared to translocate to the periphery of the cell, or even escape to the extracellular space. Clot contraction force plateaued and the generation of reactive oxygen species coincided with the initiation of platelet fragmentation, suggesting that clot contraction is stopped by the loss of cellular energy in the form of ATP due to mitochondrial dysfunction. Actin-myosin is an ATP-driven motor, and these results support the previously seen loss of the actin-regulated cytoskeleton.

The authors noted that platelet fragmentation is different from typical necrosis, as fragmentation seemed to be a regulated process that did not entail cellular rupture as the membranes remained intact. To assess whether these platelet fragments were due to apoptotic signaling, caspase activity was assessed. Surprisingly, platelets exposed to up to $5 \mathrm{U} / \mathrm{mL}$ thrombin did not appear to activate caspases. If this is not apoptosis, the question is what is responsible for platelet fragmentation? The authors identified calpain, a cysteine protease that is believed to recognize tertiary structure as a cleavage site instead of sequence-specific activity, ${ }^{10}$ as one enzyme responsible for these processes. Interestingly, maximal calpain activity coincided with the initiation of fragmentation and functional mitochondrial loss. ALLN, a calpain inhibitor, was able to delay thrombininduced fragmentation and inhibit mitochondrial loss. However, ALLN was unable to inhibit calpain cleavage products completely. Also, it is clear that inhibition of calpains alone is not enough to prevent platelet fragmentation but the observations suggest that proteases are vital for the fragmentation process to occur.

While Kim et al. provided an elegant in vitro characterization and outlined the mechanism of platelet fragmentation, the biological significance of this process awaits further elucidation. For instance, do these platelet fragments play a role in the breakdown of platelet-rich clots, and can aberrant fragmentation play a role in thrombosis? Moreover, it is worth noting the time delay in fragmentation upon treatment with thrombin and after platelet contraction, which may be significant to its function. While the GPIb-IX-V receptor complex does not typically lead to a fast and strong intracellular signal, protease-activated receptors, like most $G$ protein-coupled receptors, can rapidly induce full activation of platelets. Thus, what is the mechanism in the platelet that causes fragmentation to proceed only after platelet activation and contraction events have run their courses? The authors demonstrated that force and time are likely important factors in the process of fragmentation (Figure 1). It would be extremely interesting to understand how signaling and cytoskeletal proteins in the platelet respond to these forces and temporal factors. Furthermore, perhaps there is a balance between traditional apoptotic pathways and fragmentation during exposure to a combination of agonists. When would a platelet undergo apoptosis rather than fragmentation in response to multiple agonists? Finally, it remains to be addressed how platelet frag- 
mentation relates to platelet clearance, or what percentage of activated platelets undergo fragmentation in vivo. It is widely accepted that activated platelets are quickly cleared from the body, but the actual molecular mechanism has been elusive. Recent work on platelet clearance has focused on investigating how platelets expose a 'clear-me' signal, perhaps through desialylation of platelet surface proteins. ${ }^{11}$ The occurrence of platelet fragmentation following activation has raised the possibility that in some cases the reduction in platelet counts, which has been uniformly used as the indicator of platelet clearance, may be attributed to some extent to platelet fragmentation. It remains to be seen whether certain receptors responsible for platelet clearance can also recognize and clear platelet fragments.

If this fragmentation can be observed and tracked in vivo, perhaps the question of where fragmented platelets go after activation can be answered. A recent publication by Tomaiuolo et al. ${ }^{12}$ included high resolution images of hemostatic plugs in response to a puncture in the jugular vein. What can be gleaned from these images is the notable presence of small platelet fragments in both the intravascular and extravascular boundaries of the injury site. Determining the roles that these fragments play in hemostatic plug formation and/or thrombus formation would be crucial to a complete understanding of in vivo platelet plug formation. Potentially, this mechanism could also be a pharmacological target to reduce thrombus formation or aid in thrombolysis in pathological conditions. This exciting finding may point to a novel mechanism of platelet behavior and has major implications for thrombus dissolution and platelet clearance in general.

\section{Acknowledgments}

This work was supported in part by National Institutes of Health grant HL082808.

\section{References}

1. Dormann D, Clemetson Kj, Kehrel Be. The GPIb thrombin-binding site is essential for thrombin-induced platelet procoagulant activity. Blood. 2000;96(7):2469-2478

2. Estevez B, Kim K, Delaney Mk, et al. Signaling-mediated cooperativity between glycoprotein Ib-IX and protease-activated receptors in thrombin-induced platelet activation. Blood. 2016;127(5):626-636.

3. Tutwiler V, Litvinov Ri, Lozhkin Ap, et al. Kinetics and mechanics of clot contraction are governed by the molecular and cellular composition of the blood. Blood. 2016;127(1):149-159.

4. Kim OV, Nevzorova TA, Mordakhanova ER, et al. Fatal dysfunction and disintegration of thrombin-stimulated platelets. Haematologica. 2019;104(9):1866-1878

5. Posma IJ, Posthuma JJ, Spronk HM. Coagulation and non-coagulation effects of thrombin. J Thromb Haemost. 2016;14(10):1908-1916.

6. Bode AP, Orton SM, Frye MJ, Udis BJ. Vesiculation of platelets during in vitro aging. Blood. 1991;77(4):887-895.

7. De Paoli SH, Tegegn TZ, Elhelu OK, et al. Dissecting the biochemical architecture and morphological release pathways of the human platelet extracellular vesiculome. Cell Mol Life Sci. 2018;75(20):3781-3801.

8. Brisson AR, Tan S, Linares R, Gounou C, Arraud N. Extracellular vesicles from activated platelets: a semiquantitative cryo-electron microscopy and immuno-gold labeling study. Platelets. 2017;28(3):263271.

9. Fox JE, Boyles JK, Berndt MC, Steffen PK, Anderson LK. Identification of a membrane skeleton in platelets. J Cell Biol. 1988;106(5):1525-1538.

10. Sorimachi $\mathrm{H}$, Mamitsuka $\mathrm{H}$, Ono Y. Understanding the substrate specificity of conventional calpains. Biol Chem. 2012;393(9):853-871.

11. Quach ME, Chen W, Li R. Mechanisms of platelet clearance and translation to improve platelet storage. Blood. 2018;131(14):1512-1521.

12. Tomaiuolo M, Matzko CN, Poventud-Fuentes I, Weisel JW, Brass LF, Stalker TJ. Interrelationships between structure and function during the hemostatic response to injury. Proc Natl Acad Sci U S A. 2019;116 (6):2243-2252. 